\section{Perlecan Delineates Stem Cell Niches in Human Foetal Hip, Knee and Elbow Cartilage Rudiments and Has Potential Roles in the Regulation of Stem Cell Differentiation}

\author{
Susan M Smith ${ }^{1}$ and James Melrose ${ }^{1,2,3 *}$ \\ ${ }^{1}$ Raymond Purves Bone and Joint Research Laboratory, Kolling Institute \\ Northern Sydney Local Health District, St Leonards, Australia \\ ${ }^{2}$ Sydney Medical School, The University of Sydney, Royal North Shore \\ Hospital, Australia \\ ${ }^{3}$ Graduate School of Biomedical Engineering, University of New South \\ Wales, Sydney, Australia
}

\section{Abstract}

This study immunolocalized perlecan, Type I and II collagen and aggrecan in human foetal joint rudiment cartilages of the hip, knee and elbow. Blood vessels in the stromal tissues, associated with the cartilage rudiments were also prominently stained with perlecan, entrapped red blood cells aided in their identification. Perlecan was a prominent extracellular matrix proteoglycan in the rudiment cartilages. Stem cell niches close to the margins of the cartilage rudiments were prominently identified in the hip, knee and elbow and were distinguishable from the aforementioned blood vessels which were located in the rudiment associated stromal tissues. Type I collagen was also immunolocalized to the outer margins of the rudiment cartilages where perlecan positive niches were also present whereas the remainder of the rudiment contained type II collagen. Stem cell niches similar to those observed in the present study have previously been identified in the human foetal elbow.

Keywords: Cartilage rudiment; Human foetal joint development; Stem cell niche
\end{abstract}

\section{Introduction}

Stem cell niches similar to those observed in the present study have previously been identified in the human foetal elbow [1].

\footnotetext{
*Corresponding author: James Melrose, Raymond Purves Bone and Joint Research Laboratory, Kolling Institute Northern Sydney Local Health District, St Leonards, Australia, Tel: +61 299264806; E-mail: james.melrose@sydney.edu.au
}

Citation: Smith SM, Melrose J (2016) Perlecan Delineates Stem Cell Niches in Human Foetal Hip, Knee and Elbow Cartilage Rudiments and Has Potential Roles in the Regulation of Stem Cell Differentiation. J Stem Cell Res Dev Ther 3: 009.

Received: September 26, 2016; Accepted: November 07, 2016; Published: November 21, 2016
The aim of the present study was to determine if similar structures were also evident in hip and knee joint rudiments.

The stem cell is a specialized cell type that undergoes self-renewal to maintain a self-sustaining undifferentiated cell population within the stem cell niche [2]. The specialized microenvironment of the stem cell niche provides chemical and physical feedback cues which maintain the stem cells in a slowly recycling state of self renewal [3]. The stem cell niche acts as a reserve of cells for the replenishment of damaged or dead cells with ageing or in disease or after traumatic damage to the tissue maintaining viable cell numbers and tissue homeostasis.

Perlecan (HSPG2) is a modular proteoglycan interactive with diverse Extracellular Matrix (ECM) and cellular components [4-6]. Perlecan is a major HS substituted proteoglycan component of basement membranes and vascular tissues [7] and is also present in a vascular tissues such as articular cartilage, intervertebral disc, meniscus and tendon where it is present as a hybrid HS-CS proteoglycan [8-11]. Interactions between perlecan and structural and cell adhesive glycoproteins such as laminin, fibronectin, WARP (von Willebrand $\underline{A}$ domain-related protein), PRELP (Proline/Arginine-Rich End Leucine-Rich Repeat Protein), type IV collagen, type VI collagen, fibrillin-1, Latent Transforming Growth Factor B1 Binding Protein-2 (LTBP2), $\alpha 2 \beta 1$ and $\alpha 5 \beta 1$ integrins stabilize the ECM [12-14]. The HS chains of perlecan domain-1 bind Fibroblast Growth Factors (FGF), Vascular Endothelial Cell Growth Factor (VEGF), Platelet Derived Growth Factor (PDGF), BMP family members, Wnt (wingless-type MMTV [Mouse Mammary Tumor Virus]) integration site related proteins, hedgehog proteins regulating cellular adhesion, proliferation, differentiation, ECM synthesis, chondrogenesis and tissue morphogenesis during skeletal development [8,14-17].

Perlecan is a component of a number of stem cell niches located in the allantois, intestine, bone marrow, limbal epithelium, nervous system and elbow perichondrium [1,18-23]. Perlecan has a role in the isolation of the stem cells from ECM components outside the niche which could result in activation, migration and development of differentiated stem cell lineages [3]. FGF-2 is a major ligand for perlecan with roles in the long term viability of the niche stem cells [22,23]. Perlecan displays anti-adhesive properties to stem cells in bone marrow contributing to their isolation from extrinsic influences [24]. ECM components extrinsic to the niche are known to exert directive cues on cellular proliferation, adhesion and differentiation of stem cells [3]. Perlecan colocalises with type VI collagen pericellularly in chondrocytes and intervertebral disc cells $[25,26]$. Atomic Force Microscopy has demonstrated that perlecan provides compliancy to the type VI collagen pericellular matrix $[27,28]$ and modulates tensile stresses otherwise transmitted to cells within tensional and weight bearing connective tissues through type VI collagen [27]. Perlecan also provides an adhesive interface between the chondrocyte and its surrounding ECM [25]. The cartilage rudiments act as a transient developmental scaffold transformed by chondroprogenitor cell populations during endochondral ossification resulting in elongation of the long bones and extension of the axial skeleton [29]. 


\section{Materials and Methods}

Supplier details are as indicated in earlier studies, information on the use of the monoclonal antibodies to perlecan domain-1 (mAb A76), type I and II collagen, and aggrecan are also supplied in these publications [8-10]. Human foetal tissues (knees, elbow, hip) from one 10 week, three 12 week and four 14 week gestational age human fetuses were obtained with informed consent and all procedures were approved by our institutional ethics review committee.

\section{Results}

\section{The stem cell niche and ECM components in macroscopic} views of foetal hips

Cartilage canals were prominent features of the cartilaginous hip rudiment at 14 weeks gestational age (Figure 1). Perlecan and aggrecan were widely distributed throughout the rudiment (Figures 1a \& 1c). Type II collagen was also a prominent component of the hip rudiment in all but a surface region where type I collagen immunolocalizes (data not shown). This area was evident as a blue region in the type II immunolocalizations (Figures $1 \mathrm{~b} \& 1 \mathrm{e}$ ). Small punctate regions of high perlecan localization delineated the stem cell niches in the surface regions of the rudiment (Figure 1d) within a region devoid of type II collagen (Figure 1e) but rich in aggrecan (Figure 1f).

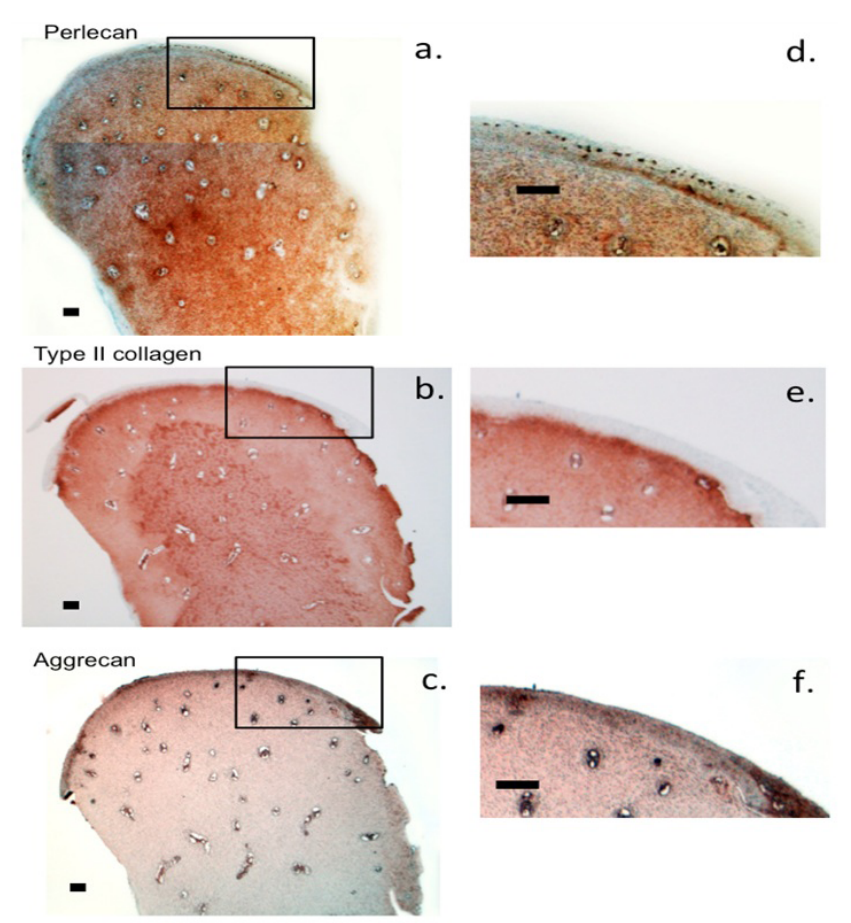

Figure 1: Immunolcalisation of perlecan (a,d), type II collagen (b,e) and aggrecan (c,f) in a 14 week old gestational age human foetal hip joint. Macrosopic views. Chromogen NovaRED, Scale bar $100 \mu \mathrm{m}$

\section{Higher power views of hip stem cell niches}

Higher magnification views of the hip rudiment perlecan immunolocalizations clearly depicted the small stem cell niches (Figures 2a2c). Cartilage canals were larger and also contained perlecan in their outer walls (Figure $2 \mathrm{~b}$ ).

\section{Stem cell niches in foetal knee rudiment cartilages}

Examination of knee rudiment cartilages also demonstrated a prominent extracellular localization of perlecan throughout the

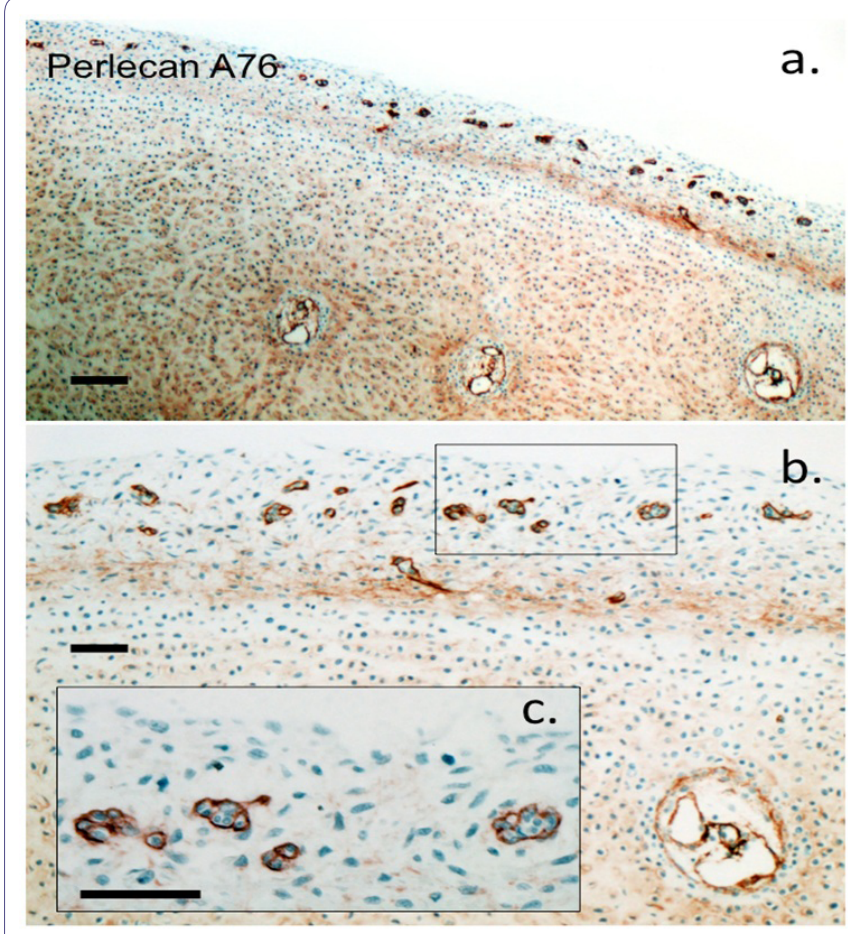

Figure 2: Higher power magnifications of the perlecan immunolocalisations depicted in figure $2 a$ using perlecan domain-1 mAb A76. The boxed area in (b) is decocted in the inset (c). Small stem cell niches are prominent in the marginal tissue of the hip cartilage rudiments. Three cartilage canals are also evident deeper in the specimen. Perlecan is also an extracellular component of the cartilage rudiment. Scale bars $100 \mu \mathrm{m}$.

femoral and tibial rudiments (Figure 3a). Small networks of cells within the surface regions of the rudiments were also prominently delineated by the perlecan immunolocalizations. We consider these are the stem cell niches and were clearly seen at higher magnification (Figures $3 \mathrm{~b} \& 3 \mathrm{c}$ ) and clearly distinguishable from blood vessels which also contain perlecan immunolocalised in the vessel wall (Figures $3 \mathrm{~d}$ \& 3e). Red blood cells were observed within some blood vessels aiding in their identification. A venule (Figure 3d) and arteriole (Figure 3e) are shown. Blood vessels were considerably larger than the stem cell stained structures (Figure $3 \mathrm{~b}$ ) which occurred in the rudiment margins whereas blood vessels were present only in the stromal tissue associated with the rudiments and did not occur in the rudiments (Figures 3d \& 3e).

Stem cell niches have characteristic morphologies and distributions in the surface region of the cartilage rudiments of joints

Closer examination of the surface regions of a number of knee joints consistently identified these niche localisations of perlecan in the surface regions of the cartilaginous rudiments (Figures $4 \mathrm{a}-4 \mathrm{~d}$ ) in a region rich in type I collagen (Figure 4e) whereas the rudiment proper contained type II collagen (Figure 4f). Aggrecan was immunolocalized diffusely throughout this outer marginal rudiment tissue zone as well as the rudiment proper (Figure $4 \mathrm{~g}$ ). While blood vessels were also prominently visualized in the stromal tissues bordering the rudiments these had a clearly differing morphology to the stem cell niche immunolocalizations of perlecan (Figure 4h). Examination of an unstained cartilage specimen by Nomarski differential interference contrast microscopy showed that cellular morphologies and arrangement of matrix components were clearly different in all of the 3 zones, stroma 


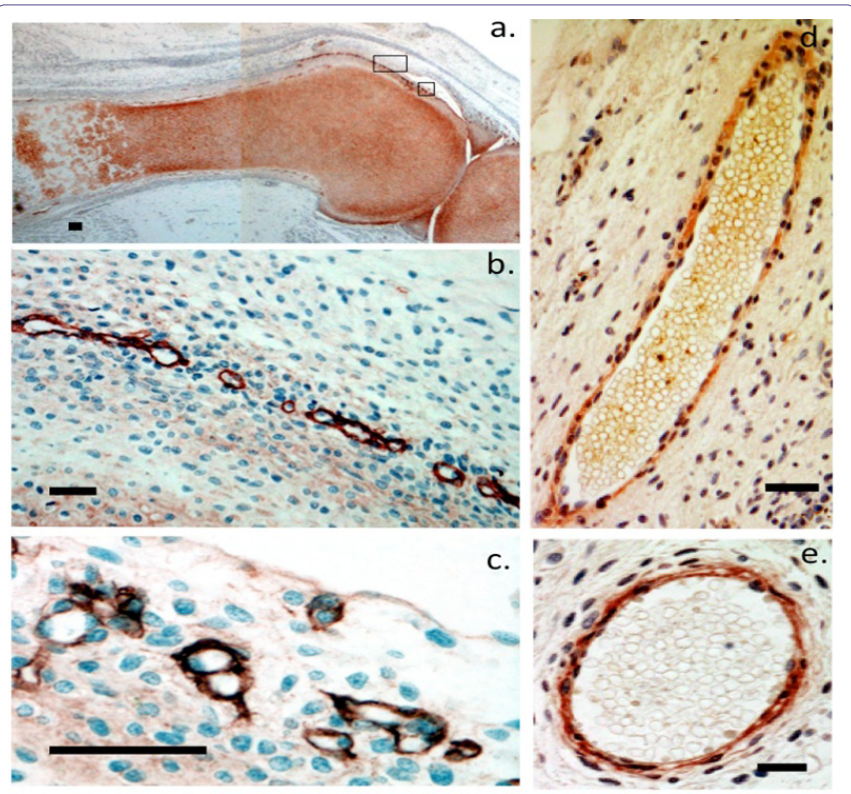

Figure 3: Immunolocalization of perlecan in a 12 week old gestational age human foetal human knee. Macroscopic view (a) and higher power magnification of areas of interest (boxed areas in (a). Stem cell niches are prominently immunolocalized $(b, c)$. Immunolocalization of perlecan delineating a venule (d) and arteriole (e) in the stromal tissue surrounding the cartilage rudiment. Red blood vessels are visible within these vessels aiding in their identification as blood vessels. Scale bar $100 \mu \mathrm{m}$.

(zone 1), rudiment margin (zone 2) and rudiment (zone 3) (Figures $4 \mathrm{i}-4 \mathrm{k})$. The cell density in zone 1 was lower than the other two zones (Figure 4i) whereas in the marginal tissue of the rudiment prominent longitudinal fibrillar material was present running parallel to the rudiment surface (Figure $4 \mathrm{j}$ ). The cells in this region had an elongated morphology (Figure 4k), type I collagen was immunolocalized to this tissue region (Figure 4e). The cells within the rudiment proper (zone 3) had a rounded morphology typical of chondrocytes and were considerably larger than the cells in the stromal tissue or rudiment margins. The cells in zone 3 were contained within lacunae similar to those seen in hyaline cartilaginous tissues.

\section{Type I collagen immunolocalizes to the surface regions of newborn hip joints}

Unfortunately we were unable to source newborn human tissue for comparison with the foetal tissue however immunolocalization of type I collagen in newborn lamb hip tissue also demonstrated it had a prominent localization in the surface region of the hip (Figure 5a) and around the cartilage canals within it. The hip tissue is cartilaginous at this stage of development. In contrast to the type I collagen immunolocalizations, type II collagen and aggrecan were immunolocalized throughout the hip rudiment (Figures $5 b$ \& $5 c$ ). Perlecan was also immunolocalized throughout the hip specimens (Figure 5d). The prominent stem cell niche arrangements so evident in the human foetal joint rudiments examined in this study were not evident in the newborn hip specimens which apparently indicates these are developmental features of human foetal joints. Immunolocalization of perlecan in a 10 week old human foetal hip rudiment showed perlecan was a prominent ECM proteoglycan, small arrangements similar to those observed in the other foetal cartilages were again present in the surface regions of the rudiments however the humeral head had not completely separated from the adjacent acetabulum at this stage of joint development (Figure 5e). Such perlecan positive arrangements

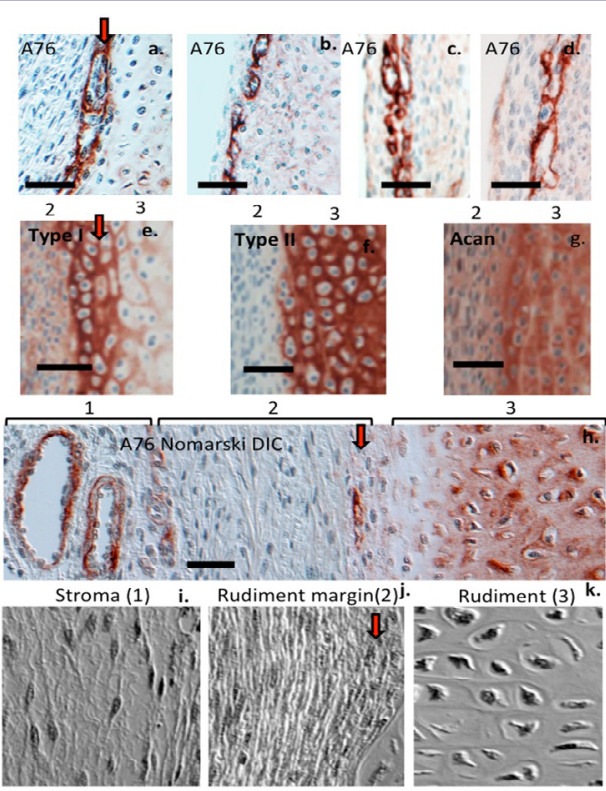

Figure 4: Immunolocalization of stem cell niches using mAb A76 to perlecan domain-1 in the outer margins of a tibial cartilaginous rudiment (12 week gestational age) (a-d) and type I collagen (e) type II collagen (f) and aggrecan (Acan) (g) in the cartilaginous rudiment (zone 3 ) and rudiment marginal tissue (zone 2). Immunolocalization of two prominent blood vessels in the stromal tissue (zone 1) adjacent to the rudiment (zones 2,3). Perlecan is also an extracellular matrix component in zone $3(\mathrm{~h})$. Unstained black and white Nomarski phase contrast DIC images of representative areas of zone 1-3 clearly identifying differences in cellular morphology and matrix organization in these areas (i-k).

were also observed in a 12 week old human foetal elbow rudiment (Figure $5 \mathrm{f}$ ) in the surface regions of the cartilage rudiments (Figures $5 g \& 5 h)$.
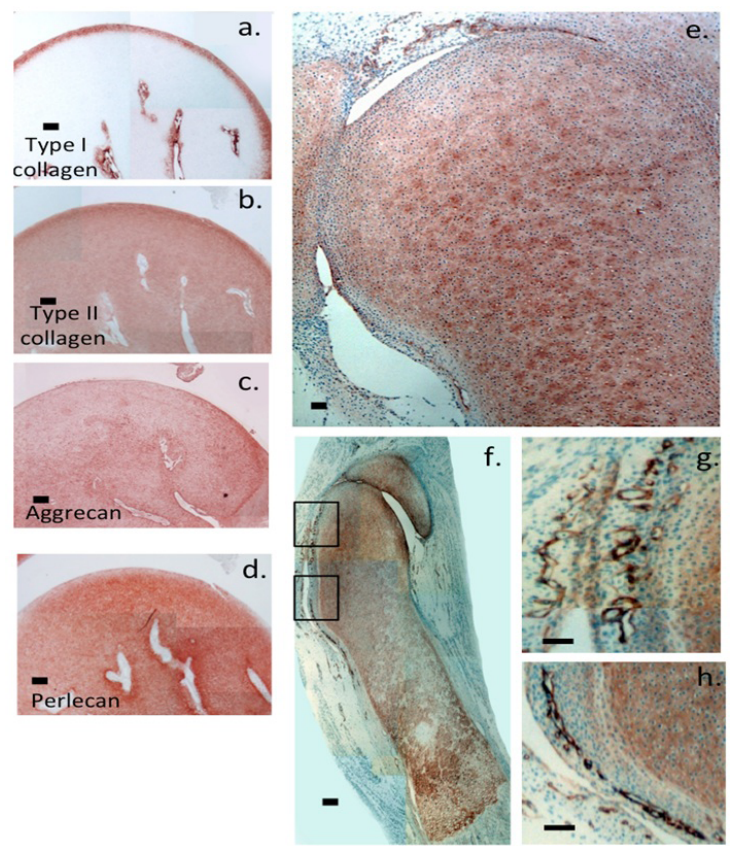

Figure 5: Macroscopic immunolocalization of type I collagen (a), type II collagen (b) aggrecan (c) and perlecan (d) in a newborn ovine hip joint and 10 week gestational age human foetal hip joint which has only partially separated from the acetabulum (e) and a 12 week old human foetal elbow (f). Cartilage canals are prominent in (a-d). Small stem cell niches are evident in the marginal rudiment tissues of (e and f). NovaRED chromogen, Scale bars $100 \mu \mathrm{m}$. 


\section{Discussion}

The present study demonstrated roles for perlecan in foetal human joint development not only as an ECM component in the cartilaginous rudiments but also as a stem cell niche component with potential roles in the regulation of stem cell differentiation. This is in keeping with perlecans known roles in chondrogenesis [8] and the regulation of cellular adhesion and proliferation in skeletal development $[5,15,16,30]$. The complexity of the dynamic native stem cell niche microenvironment is a function of the surface biochemistry, topography (type, size, organization, and geometry of nanostructures), and their mechanical properties determining whether the stem cell remains in a quiescent non-differentiated state or otherwise. Signalling pathways involving Notch, Wingless-type (Wnt), Sonic hedgehog (Shh), and Smad proteins are important determinants of self-renewal and differentiation of stem cells. Type I collagen is a component of many stem cell niches $[31,32]$ and may provide an interactive mechanical and biochemical niche environment conducive to stem cell differentiation, adhesion and migration [33-38]. MSCs sense a 3D environment containing type I collagen through discoid in domain receptor-1 [31]. The localization of type I collagen around the stem cell niches identified in the present study may therefore be important for stem cell differentiation and the morphogenesis of rudiment cartilage to bone [29]. Type I collagen occurs in tissues to counter tensional forces, the tensional properties of growth surfaces have been shown to be an important modulator of stem cell behavior. Perlecan colocalises with collagen networks in the pericellular matrix of cells providing compliancy properties to tissues thus the localization of perlecan in the stem cell niche may counter the tensile properties conveyed by the type I collagen outside the niche. Atomic Force Microscopy (AFM) of single hip chondrocytesin a mouse model of Schwartz Jampel syndrome demonstrated a significant effect on cell and tissue stiffness implicating outside-in mechanical signalling from the pericellular matrix as a dynamic stimulant to cellular development [39]. A similar scenario may also apply to the regulation of stem cells in the perlecan niches observed in the present study. While we refer to these as stem cell niches throughout this manuscript these should more correctly be considered "putative" stem cell niches based on the data we have presented in the present study. Unfortunately we were not able to undertake immunolocalizations with other antibodies to confirm the identity of these perlecan positive structures and this is a weakness of our study. However we considered the observations we made were nevertheless important enough to expound such an interpretation and in our opinion terming these as niches fitted a number of criteria proposed in other studies as to what constitutes a niche. Further studies should aim to rectify this deficiency.

The Wnt family of growth factors are ancient metazoan proteins which are conserved throughout vertebrate and invertebrate evolution. Wnt proteins (the name Wnt is a fusion of the name for the Drosophila segment polarity gene 'wingless' and its vertebrate homolog, 'integrated or int-1') regulate stem cell fate, migration, proliferation and self-renewal [40-44]. Extracellular Wnt protein induces a number of intracellular signal transduction pathways of importance in stem cell differentiation, tissue development and repair [40,41,43-45]. Perlecan regulates bi-directional Wnt signaling in Drosophila [40,46-49] and growth factor signaling in C.elegans [50] and also stabilizes the matrix surrounding the stem cell niche. Recent studies in the human foetal elbow showed that perlecan localized with type I collagen surrounding the stem cell niches [1]. Type I collagen normally conveys tensile forces in tissues, AFM studies have shown that perlecan provided compliancy to the type VI collagen pericellular matrix surrounding chondrocytes where these components were co-localized as in IVD cells $[25,27,28]$. Thus the perlecan, which delineates the stem cell niches observed in the present study, may modulate the propagation of tensile forces to the niche stem cells from their external micro-environment. Knockdown of perlecan lowers in situ cell and matrix stiffness in developing cartilage [39]. The stem cell microenvironment is influenced by matrix stiffness, and mechanical forces experienced from fluid shear, compression and tension, and these regulate the proliferation and differentiation of stem cells [51-54]. MSCs sense biomechanical forces through primary cilia which are colocalised with calcium ion channels resulting in responsive changes in mechanosensitive ion channel associated proteins such as transient receptor potential melastatin 7 , a mechanosensitive plasma membrane calcium channel protein, and changes in their cytoskeleton [54]. An influx of $\mathrm{Ca}^{2+}$ ions into MSCs has attendant effects on the formation of intracellular signaling molecules such as inositol triphosphate, changes in the actin/actomyosin cytoskeleton and the activation of associated signaling pathways. The associated change in cell shape and co-ordination of focal adhesions acts as a primer for MSC migration. MSCs are also capable of secreting active heparanase- 1 which degrades the HS side chains of perlecan [55]. Heparanase released from MSCs also activates Integrin beta1/HIF-2alpha/Flk-1 signaling and stem cell migration $[55,56]$. Many stem cell populations utilize the SDF-1/CXCR4 axis to effect migration [57-61]. The release of progenitor cell populations from their niches by heparanase- 1 is consistent with emerging roles for heparanase- 1 and 2 in the promotion of wound repair [62,63]. Live cell imaging of stem cell niches where the niche is de-stressed results in a change in the stem cell quiescent state to a migratory phenotype which can home to sites of tissue damage [64,65]. This is consistent with roles proposed for biomechanical forces and ECM directive cues in the regulation of stem cell phenotype in vivo and the promotion of wound healing [3,66-69].

The stem cell arrangements visualized in the present study were present in the rudiment margins and were clearly distinguishable from blood vessels in the stromal tissues. Like other cartilages, the rudiments contain anti-angiogenic proteins refractory to the penetration of blood vessels $[14,70]$. An earlier confocal study of the human foetal elbow demonstrated perlecan positive niches in the outer regions of the perichondrium along the shaft of the long bones [1]. Activated stem cells displaying the CS sulphation motifs $4 \mathrm{C} 3,3 \mathrm{~B} 3(-)$ and 7D4 were closely associated with these perlecan positive niches [1]. Chondroprogenitor stem cells have also been identified in the surface regions of the presumptive articular cartilage of the knee joint following joint cavitation [71,72].

With an increased awareness of the role of extrinsic forces on stem cell regulation in situ [3], regenerative approaches are now being developed using bio-scaffolds with defined tunable tensional properties to modulate stem cell differentiation in vitro [68]. Decellularised tissues with their ECM, biochemical and structural cues intact in the stem cell niche are also being evaluated for their ability to modulate stem cell behaviour in culture and modify stem cell proliferation and differentiation [73]. The stiffness of biomaterials is an important determinant which drives stem cell proliferation and differentiation [74]. Evaluation of matrices synthesized by human fibroblasts has shown an increase in stem cell proliferation and differentiation, down-regulation of adipogenesis and osteogenesis but promotion of chondrogenesis [69]. In the human foetal elbow the perichondrial stem cell niche is contained in a matrix layed down by fibroblastic cells in the outer 
Citation: Smith SM, Melrose J (2016) Perlecan Delineates Stem Cell Niches in Human Foetal Hip, Knee and Elbow Cartilage Rudiments and Has Potential Roles in the Regulation of Stem Cell Differentiation. J Stem Cell Res Dev Ther 3: 009.

regions of the perichondrium. These regions of the perichondrium are rich in type I collagen [1] and are similar to the areas of type I collagen deposition between the rudiment and associated stromal tissues observed in the present study and which also contain stem cell niches.

Matricryptins and matrikines have received considerable attention as prospective agents for tissue repair through their ability to modify stem cell behavior stimulating proliferation and differentiation [70], some matrikines stimulate stem cell migration. Chondroitin Sulphate (CS) is another Glycosaminoglycan (GAG) which is considered indispensable for stem cell pluripotency and stem cell differentiation [75], We are now in an exciting era in regenerative medicine and may be optimistic that armed with greater knowledge on how stem cells are regulated in their niches we may someday be able to manipulate these to improve their replicative, differentiative and migratory properties in situ to obtain the critical numbers of stem cells of specific cell lineages required at defect sites for regenerative applications. It is to be expected that a greater understanding of how stem cells target damaged tissues will also greatly improve the utility of stem cells as therapeutic agents.

\section{Acknowledgement}

This study was supported by NHMRC Project Grant 1004032.

\section{Author Contributions}

Susan M Smith undertook the immunolocalizations presented in this study, reviewed the final version of the manuscript and assisted in writing the methods section. James Melrose obtained funding, conceptualized the study, collected and interpreted data, prepared figures and wrote the manuscript.

\section{References}

1. Hayes AJ, Hughes CE, Smith SM, Caterson B, Little CB, et al. (2016) The CS Sulfation Motifs 4C3, 7D4, 3B3[-]; and Perlecan Identify Stem Cell Populations and Their Niches, Activated Progenitor Cells and Transitional Areas of Tissue Development in the Fetal Human Elbow. Stem Cells Dev 25: 836-847.

2. Guest I, llic Z, Sell S (2016) Origin of the stem cell niche concept. Exp Hematol 44: 809-810.

3. Ahmed M, Ffrench Constant C (2016) Extracellular Matrix Regulation of Stem Cell Behavior. Curr Stem Cell Rep 2: 197-206.

4. Farach Carson MC, Carson DD (2007) Perlecan--a multifunctional extracellular proteoglycan scaffold. Glycobiology 17: 897-905.

5. Knox SM, Whitelock JM (2006) Perlecan: how does one molecule do so many things?. Cell Mol Life Sci 63: 2435-2445.

6. Melrose J, Hayes AJ, Whitelock JM, Little CB (2008) Perlecan, the "jack of all trades" proteoglycan of cartilaginous weight-bearing connective tissues. Bioessays 30: 457-469.

7. Iozzo RV, Cohen IR, Grassel S, Murdoch AD (1994) The biology of perlecan the multifaceted heparan sulphate proteoglycan of basement membranes and pericellular matrices. Biochem J 302: 625-639.

8. Smith SM, Shu C, Melrose J (2010) Comparative immunolocalisation of perlecan with collagen II and aggrecan in human foetal, newborn and adult ovine joint tissues demonstrates perlecan as an early developmental chondrogenic marker. Histochem Cell Biol 134: 251-263.

9. Smith SM, Whitelock JM, lozzo RV, Little CB, Melrose J, et al. (2009) Topographical variation in the distributions of versican, aggrecan and perlecan in the foetal human spine reflects their diverse functional roles in spinal development. Histochem Cell Biol 132: 491-503.

10. Melrose J, Smith S, Cake M, Read R, Whitelock J, et al. (2005) Comparative spatial and temporal localisation of perlecan, aggrecan and type I, II and IV collagen in the ovine meniscus: an ageing study. Histochem Cell Biol 124: 225-235.
11. Melrose J, Smith S, Cake M, Read R, Whitelock J, et al. (2005) Perlecan displays variable spatial and temporal immunolocalisation patterns in the articular and growth plate cartilages of the ovine stifle joint. Histochem Cell Biol 123: $561-571$.

12. Hayes AJ, Gibson MA, Shu C, Melrose J (2014) Confocal microscopy demonstrates association of LTBP-2 in fibrillin-1 microfibrils and colocalisation with perlecan in the disc cell pericellular matrix. Tissue Cell 46: 185-197.

13. Hayes AJ, Smith SM, Gibson MA, Melrose J (2011) Comparative immunolocalization of the elastin fiber-associated proteins fibrillin-1, LTBP-2, and MAGP-1 with components of the collagenous and proteoglycan matrix of the fetal human intervertebral disc. Spine (Phila Pa 1976) 36: 1365-1372.

14. Whitelock JM, Melrose J, lozzo RV (2008) Diverse cell signaling events modulated by perlecan. Biochemistry 47: 11174-11183.

15. Lord MS, Chuang CY, Melrose J, Davies MJ, lozzo RV, et al. (2014) The role of vascular-derived perlecan in modulating cell adhesion, proliferation and growth factor signaling. Matrix Biol 35: 112-122.

16. Farach Carson MC, Brown AJ, Lynam M, Safran JB, Carson DD, et al. (2008) A novel peptide sequence in perlecan domain IV supports cell adhesion, spreading and FAK activation. Matrix Biol 27: 150-160.

17. Nakamura R, Nakamura F, Fukunaga S (2015) Perlecan Diversely Regulates the Migration and Proliferation of Distinct Cell Types in vitro. Cells Tissues Organs 200: 374-393.

18. Mikedis MM, Downs KM (2009) Collagen type IV and Perlecan exhibit dynamic localization in the Allantoic Core Domain, a putative stem cell niche in the murine allantois. Dev Dyn 238: 3193-3204.

19. You J, Zhang Y, Li Z, Lou Z, Jin L, et al. (2014) Drosophila perlecan regulates intestinal stem cell activity via cell-matrix attachment. Stem Cell Reports 2: 761-769.

20. Schofield KP, Gallagher JT, David G (1999) Expression of proteoglycan core proteins in human bone marrow stroma. Biochem J 343: 663-668.

21. Schlotzer Schrehardt U, Dietrich T, Saito K, Sorokin L, Sasaki T, et al. (2007) Characterization of extracellular matrix components in the limbal epithelial stem cell compartment. Exp Eye Res 85: 845-860.

22. Kerever A, Mercier F, Nonaka R, de Vega S, Oda Y, et al. (2014) Perlecan is required for FGF-2 signaling in the neural stem cell niche. Stem Cell Res 12: $492-505$

23. Kerever A, Schnack J, Vellinga D, Ichikawa N, Moon C, et al. (2007) Novel extracellular matrix structures in the neural stem cell niche capture the neurogenic factor fibroblast growth factor 2 from the extracellular milieu. Stem Cells 25: 2146-2157.

24. Klein G, Conzelmann S, Beck S, Timpl R, Müller CA (1995) Perlecan in human bone marrow: a growth-factor-presenting, but anti-adhesive, extracellular matrix component for hematopoietic cells. Matrix Biol 14: 457-465.

25. Hayes AJ, Shu CC, Lord MS, Little CB, Whitelock JM, et al. (2016) Pericellular colocalisation and interactive properties of type $\mathrm{VI}$ collagen and perlecan in the intervertebral disc. Eur Cell Mater 32: 40-57.

26. Zelenski NA, Leddy HA, Sanchez-Adams J, Zhang J, Bonaldo P, et al. (2015) Type VI Collagen Regulates Pericellular Matrix Properties, Chondrocyte Swelling, and Mechanotransduction in Mouse Articular Cartilage. Arthritis Rheumatol 67: 1286-1294.

27. Wilusz RE, Defrate LE, Guilak F (2012) A biomechanical role for perlecan in the pericellular matrix of articular cartilage. Matrix Biol 31: 320-327.

28. Wilusz RE, Sanchez-Adams J, Guilak F (2014) The structure and function of the pericellular matrix of articular cartilage. Matrix Biol 39: 25-32.

29. Melrose J, Shu C, Whitelock J M, Lord MS (2016) The cartilage extracellular matrix as a transient developmental scaffold for growth plate maturation. Matrix Biol 52-54: 363-383.

30. lozzo RV (1994) Perlecan: a gem of a proteoglycan. Matrix Biol 14: 203-208.

31. Lund AW, Stegemann JP, Plopper GE (2009) Mesenchymal Stem Cells Sense Three Dimensional Type I Collagen through Discoidin Domain Receptor 1. Open Stem Cell J 1: 40-53. 
Citation: Smith SM, Melrose J (2016) Perlecan Delineates Stem Cell Niches in Human Foetal Hip, Knee and Elbow Cartilage Rudiments and Has Potential Roles in the Regulation of Stem Cell Differentiation. J Stem Cell Res Dev Ther 3: 009.

32. Motegi H, Kamoshima Y, Terasaka S, Kobayashi H, Houkin K (2014) Type 1 collagen as a potential niche component for CD133-positive glioblastoma cells. Neuropathology 34: 378-385.

33. Bergström T, Holmqvist $\mathrm{K}$, Tararuk $\mathrm{T}$, Johansson $\mathrm{S}$, Forsberg-Nilsson $\mathrm{K}$ (2014) Developmentally regulated collagen/integrin interactions confer adhesive properties to early postnatal neural stem cells. Biochim Biophys Acta 1840: 2526-2532.

34. Di Felice V, Ardizzone NM, De Luca A, Marcianò V, Marino Gammazza A, et al. (2009) OPLA scaffold, collagen I, and horse serum induce an higher degree of myogenic differentiation of adult rat cardiac stem cells. J Cell Physiol 221: 729-739.

35. Herrera-Perez M, Voytik-Harbin SL, Rickus JL (2015) Extracellular Matrix Properties Regulate the Migratory Response of Glioblastoma Stem Cells in Three-Dimensional Culture. Tissue Eng Part A 21: 2572-2582.

36. Kruger EA, Im DD, Bischoff DS, Pereira CT, Huang W, et al. (2011) In vitro mineralization of human mesenchymal stem cells on three-dimensional type I collagen versus PLGA scaffolds: a comparative analysis. Plast Reconstr Surg 127: 2301-2311.

37. Nii M, Lai JH, Keeney M, Han LH, Behn A, et al. (2013) The effects of interactive mechanical and biochemical niche signaling on osteogenic differentiation of adipose-derived stem cells using combinatorial hydrogels. Acta Biomater 9: $5475-5483$

38. Takebayashi T, Horii T, Denno H, Nakamachi N, Otomo K, et al. (2013) Human mesenchymal stem cells differentiate to epithelial cells when cultured on thick collagen gel. Biomed Mater Eng 23: 143-153.

39. Xu X, Li Z, Leng Y, Neu CP, Calve S (2016) Knockdown of the pericellular matrix molecule perlecan lowers in situ cell and matrix stiffness in developing cartilage. Developmental Biology 418: 242-247.

40. Komiya Y, Habas R (2008) Wnt signal transduction pathways. Organogenesis 4: 68-75.

41. Mohammed MK, Shao C, Wang J, Wei Q, Wang X, et al. (2016) Wnt/B -catenin signaling plays an ever-expanding role in stem cell self-renewal, tumorigenesis and cancer chemoresistance. Genes Dis 3: 11-40.

42. Nava MM, Raimondi MT, Pietrabissa R (2012) Controlling self-renewal and differentiation of stem cells via mechanical cues. Biomed Res Int 2012: 1-12.

43. Nusse R (2008) Wnt signaling and stem cell control. Cell Res 18: 523-527.

44. Nusse R, Fuerer C, Ching W, Harnish K, Logan C, et al. (2008) Wnt signaling and stem cell control. Cold Spring Harb Symp Quant Biol 73: 59-66.

45. Yang Y (2012) Wnt signaling in development and disease. Cell Biosci 2: 14.

46. Kamimura K, Ueno K, Nakagawa J, Hamada R, Saitoe M, et al. (2013) Perlecan regulates bidirectional Wnt signaling at the Drosophila neuromuscular junction. J Cell Biol 200: 219-233.

47. Lane SW, Williams DA, Watt FM (2014) Modulating the stem cell niche for tissue regeneration. Nat Biotechnol 32: 795-803.

48. Lindner JR, Hillman PR, Barrett AL, Jackson MC, Perry TL, et al. (2007) The Drosophila Perlecan gene trol regulates multiple signaling pathways in different developmental contexts. BMC Dev Biol 7: 121.

49. Matsuo I, Kimura-Yoshida C (2014) Extracellular distribution of diffusible growth factors controlled by heparan sulfate proteoglycans during mammalian embryogenesis. Philos Trans R Soc Lond B Biol Sci 369.

50. Merz DC, Alves G, Kawano T, Zheng H, Culotti JG (2003) UNC-52/perlecan affects gonadal leader cell migrations in $\mathrm{C}$. elegans hermaphrodites through alterations in growth factor signaling. Dev Biol 256: 173-186.

51. Bhattacharyya S, Kumar A, Lal Khanduja K (2012) The voyage of stem cel toward terminal differentiation: a brief overview. Acta Biochim Biophys Sin (Shanghai) 44: 463-475.

52. Janmey PA, Miller RT (2011) Mechanisms of mechanical signaling in development and disease. J Cell Sci 124: 9-18.
53. Liu YS, Lee OK (2014) In search of the pivot point of mechanotransduction: mechanosensing of stem cells. Cell Transplant 23: 1-11.

54. Yim EK, Sheetz MP (2012) Force-dependent cell signaling in stem cell differentiation. Stem Cell Res Ther 3: 41.

55. Hu X, Zhang L, Jin J, Zhu W, Xu Y, et al. (2015) Heparanase released from mesenchymal stem cells activates integrin beta1/HIF-2alpha/Flk-1 signaling and promotes endothelial cell migration and angiogenesis. Stem Cells 33: 1850-1862.

56. Cheng CC, Lee YH, Lin SP, Huangfu WC, Liu IH (2014) Cell-autonomous heparanase modulates self-renewal and migration in bone marrow-derived mesenchymal stem cells. J Biomed Sci 21: 21.

57. Li J, Guo W, Xiong M, Han H, Chen J, et al. (2015) Effect of SDF-1/CXCR4 axis on the migration of transplanted bone mesenchymal stem cells mobilized by erythropoietin toward lesion sites following spinal cord injury. Int J Mol Med 36: $1205-1214$

58. Tu TC, Nagano M, Yamashita T, Hamada H, Ohneda K, et al. (2016) A Chemokine Receptor, CXCR4, Which Is Regulated by Hypoxia-Inducible Factor $2 \alpha$, Is Crucial for Functional Endothelial Progenitor Cells Migration to Ischemic Tissue and Wound Repair. Stem Cells Dev 25: 266-276.

59. Xue L, Wang J, Wang W, Yang Z, Hu Z, et al. (2014) The effect of stromal cell-derived factor 1 in the migration of neural stem cells. Cell Biochem Biophys 70: 1609-1616.

60. Yu Y, Wu RX, Gao LN, Xia Y, Tang HN, et al. (2016) Stromal cell-derived factor-1-directed bone marrow mesenchymal stem cell migration in response to inflammatory and/or hypoxic stimuli. Cell Adh Migr 10: 342-359.

61. Zhao $Y$, Zhang $H$ (2016) Update on the mechanisms of homing of adipose tissue-derived stem cells. Cytotherapy 18: 816-827.

62. Nasser NJ (2008) Heparanase involvement in physiology and disease. Cell Mol Life Sci. 65: 1706-1715.

63. Zcharia E, Zilka R, Yaar A, Yacoby-Zeevi O, Zetser, et al. (2005) Heparanase accelerates wound angiogenesis and wound healing in mouse and rat models. FASEB J 19: 211-221

64. Park S, GrecoV, Cockburn K (2016) Live imaging of stem cells: answering old questions and raising new ones. Curr Opin Cell Biol. 43: 30-37.

65. Yu NY, O'Brien CA, Slapetova I, Whan RM, Knothe Tate ML (2016) Live Tissue Imaging to Elucidate Mechanical Modulation of Stem Cell Niche Quiescence. Stem Cells Transl Med.

66. Daley WP, Peters SB, Larsen M (2008) Extracellular matrix dynamics in development and regenerative medicine. J Cell Sci 121: 255-264.

67. Kim SH, Turnbull J, Guimond S (2011) Extracellular matrix and cell signalling: the dynamic cooperation of integrin, proteoglycan and growth factor receptor. J Endocrinol 209: 139-151.

68. Subbiah R, Hwang MP, Du P, Suhaeri M, Hwang JH, et al. (2016) Tunable Crosslinked Cell-Derived Extracellular Matrix Guides Cell Fate. Macromol Biosci.

69. Zhou Y, Zimber M, Yuan H, Naughton GK, Fernan R, et al. (2016) Effects of Human Fibroblast-Derived Extracellular Matrix on Mesenchymal Stem Cells. Stem Cell Rev 12: 560-572.

70. Ricard Blum S, Ballut $L$ (2011) Matricryptins derived from collagens and proteoglycans. Front Biosci (Landmark Ed) 16: 674-697.

71. Archer CW, Dowthwaite GP, Francis West P (2003) Development of synovial joints. Birth Defects Res C Embryo Today 69:144-155.

72. Dowthwaite GP, Bishop JC, Redman SN, Khan IM, Rooney P, et al. (2004) The surface of articular cartilage contains a progenitor cell population. J Cell Sci. 117: 889-897.

73. Evans ND, Minelli C, Gentleman E, LaPointe V, Patankar, et al. (2009) Substrate stiffness affects early differentiation events in embryonic stem cells. Eur Cell Mater 18: 1-13 
Citation: Smith SM, Melrose J (2016) Perlecan Delineates Stem Cell Niches in Human Foetal Hip, Knee and Elbow Cartilage Rudiments and Has Potential Roles in the Regulation of Stem Cell Differentiation. J Stem Cell Res Dev Ther 3: 009.

- Page 7 of 7 •

74. Roberts JN, Sahoo JK, McNamara LE, Burgess KV, Yang J, et al. (2016) Dynamic Surfaces for the Study of Mesenchymal Stem Cell Growth through Adhesion Regulation. ACS Nano 10: 6667-6679.
75. Izumikawa T, Sato B, Kitagawa $\mathrm{H}(2014)$ Chondroitin sulfate is indispensable for pluripotency and differentiation of mouse embryonic stem cells. Sci Rep 4: 3701. 